\title{
Numerical simulations of the Richtmyer-Meshkov instability with reshock
}

\author{
Pooya Movahed ${ }^{1}$ and Eric Johnsen ${ }^{2}$ \\ University of Michigan, Ann Arbor, MI, 48109-2133
}

\begin{abstract}
Two-dimensional simulations of the Richtmyer-Meshkov instability with re-shock are carried out based on the single-mode Mach 1.21 air/ $/ \mathrm{SF}_{6}$ shock tube experiments of Collins and Jacobs. A second-order accurate MUSCL-Hancock scheme and several high-order WENO schemes are used for shock capturing along with a gamma-based model for interface capturing to allow for the stable and accurate representation of fluids of different ratios of specific heats. The present results are compared qualitatively and quantitatively with existing theoretical models, experimental data and computational results. Good quantitative agreement with the observed amplitude growth, interface velocity and time of reshock are achieved. The amount and role of numerical dissipation of different schemes on the physical properties of the flow such as the circulation, enstrophy, mixing and kinetic energy of the small scales are also investigated.
\end{abstract}

\section{Nomenclature}

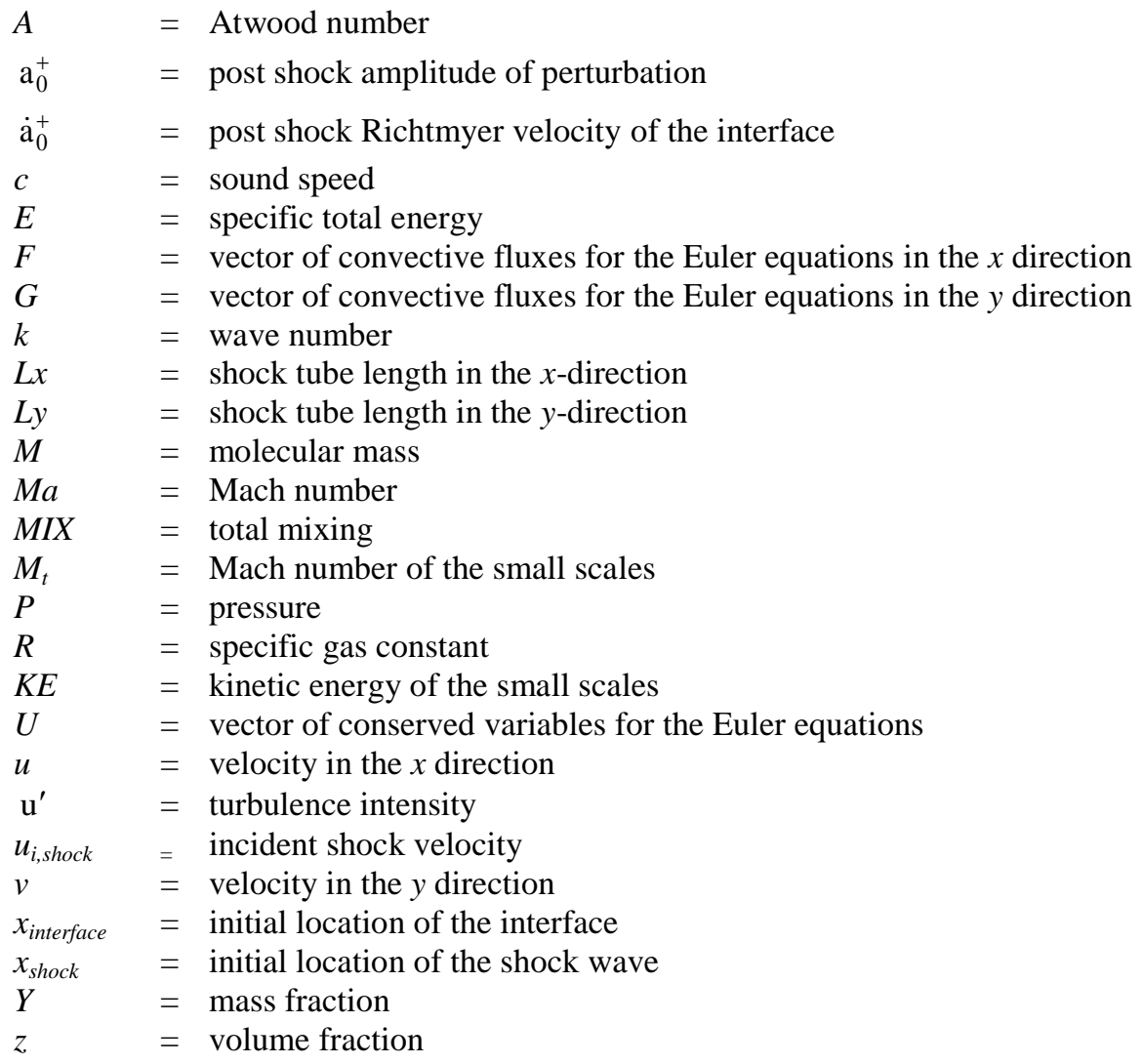

${ }^{1}$ Graduate Student Research Assistant, Department of Mechanical Engineering, 1231 Beal Ave., 2043 Walter E. Lay Automotive Laboratory, Ann Arbor, MI 48109-2133, AIAA Member.

${ }^{2}$ Assistant Professor, Department of Mechanical Engineering, 1231 Beal Ave., 2043 Walter E. Lay Automotive Laboratory, Ann Arbor, MI 48109-2133, AIAA Member.

American Institute of Aeronautics and Astronautics 


$\begin{array}{ll}\Gamma & =\text { circulation } \\ \Gamma^{\prime} & =\text { circulation deposited per unit length of an unshocked planar interface } \\ \Delta u & =\text { velocity of the unperturbed interface after interacting with the incident shock wave } \\ \Omega & =\text { vorticity distribution function } \\ \gamma & =\text { specific heat ratio } \\ \eta & =\text { density ratio } \\ \lambda & =\text { wave length } \\ \rho & =\text { density } \\ \omega & =\text { vorticity vector } \\ E N S & =\text { enstrophy }\end{array}$

\section{Introduction}

$\mathrm{T}$ HE Richtmyer-Meshkov (RM) instability occurs when a shock interacts with a perturbed interface separating fluids of different densities. ${ }^{1,2}$ The misalignment of the density gradient across the interface and the pressure gradient across the shock wave leads to baroclinic vorticity generation along the interface which is the basic mechanism for the amplification of any perturbations initially present along the interface. ${ }^{3}$ The vorticity evolution equation for the two-dimensional compressible inviscid flows simplifies to

$$
\frac{D \omega}{D t}=\frac{1}{\rho^{2}}(\nabla \rho \times \nabla P)-w \nabla . u
$$

where $\omega=\nabla \times u$ is the vorticity vector normal to the plane of the motion. The shock wave initially compresses the perturbations existing along the interface. Shortly after the passage of the shock, a vortex sheet is created by the baroclinic vorticity (first term on the right-hand side of Eq. 1) and induces different velocities at each point relative to the unperturbed interface. The relative motion along the interface causes the lighter gas to penetrate into the heavier gas, thus forming a bubble, and the heavier gas to rise into the lighter gas and roll-up forming a spike. The heavy/light configuration, which is stable in the Rayleigh-Taylor instability, is also unstable in the RM instability and the amplitude will grow after a phase inversion. The secondary instabilities, such as the Rayleigh-Taylor and the Kelvin-Helmholtz instabilities also occur at later times and help the roll-up process at spike leading to the appearance of mushroom-like structures. ${ }^{4}$

There are different applications for the RM instability at different scales. At large scales, the observed early appearance of heavy elements (like $\mathrm{Ni}$ and $\mathrm{Co}$ ) in the photosphere of supernova 1987A indicates that the RM and Rayleigh-Taylor instabilities have significant and observable effect. ${ }^{5}$ The RM instability also occurs in supersonic combustion applications affecting the mixing rate of the air and fuel in ramjet engines. ${ }^{6}$ At small scales, the RM instability plays a dominant role in the implosion of the deuterium-tritium capsule in Inertial Confinement Fusion and reduces the efficiency by mixing the ablator with the fuel. ${ }^{7}$ The hydrodynamic instabilities occurring in systems that obey the Euler equations are invariant under scale transformation ranging from astrophysical applications to small-scales instabilities in Inertial Confinement Fusion. ${ }^{8}$ Although cylindrical and spherical configurations are more representative of the applications mentioned above, most of the work in the literature has been performed in a planar geometry with single or multimode perturbations for simplicity. In this work, we have also chosen scales similar to those of planar configuration shock tube experiments to perform our numerical investigations.

In the planar case, the geometry may be such that, after the shock has passed through the interface, it reflects off the end wall and impinge upon the distorted interface again (reshock). Complex small-scale features appear right after reshock and enhance the mixing rate. Secondary baroclinic vorticity is generated and affects the small scale features responsible for the increase in circulation on each side of the interface. ${ }^{9}$ The mixing region eventually becomes turbulent and molecularly mixed at late times. Vortex stretching effects that are not present in twodimensional simulations may have dominant role in the interface behavior especially after reshock when the flow becomes turbulent. Due to limitations of the computing facilities, three-dimensional simulations of the RM instability are not still practical for Inertial Confinement Fusion design. Recently, there have been some efforts to understand the turbulence behavior of the three-dimensional RM instability by using large-eddy simulation. ${ }^{10}$

In this work, we have carried out a set of simulations using a second-order MUSCL-Hancock scheme and several high-order WENO shock capturing schemes to investigate the single-mode Richtmyer-Meshkov instability with reshock. ${ }^{11-15}$ The interface-capturing method used in this work enables us to model interfaces separating fluids of different specific heats ratio without generating spurious pressure oscillations at the interface. The initial conditions correspond to the Collins and Jacobs experiment. ${ }^{16}$ The amplitude growth rates for different schemes 
show good agreement with the experiment specially before the reshock for all of the schemes on the resolutions used here and are also compared with analytical models. The effects of resolution and numerical dissipation of the different schemes on the physical properties of the flow are discussed in detail both qualitatively and quantitatively.

\section{Governing Equations}

The two-dimensional inviscid Euler equations are solved in conservative form

$$
U_{t}+F_{x}+G_{y}=0
$$

where the conserved variables $U$ and convective fluxes $F$ and $G$ are

$$
U=(\rho, \rho u, \rho v, \rho E)^{T}, F=\left(\rho u, \rho u^{2}+P, \rho u v, u(\rho E+P)\right)^{T}, G=\left(\rho v, \rho u v, \rho v^{2}+P, v(\rho E+P)\right)^{T}
$$

Here, $\rho$ is density, $P$ is pressure, $(u, v)$ is the velocity vector, $E=\left(u^{2}+v^{2}\right) / 2+P /(\rho(\gamma-1))$ is the total energy per unit volume. The ideal gas equation $\mathrm{P}=\rho \mathrm{RT}$ is used as the equation of state.

For multicomponent problems, we need to solve an additional equation describing the fluid composition to complete our set of equations. In order to prevent spurious pressure oscillations at interfaces, a $\gamma$-based model is used. ${ }^{17,18}$ In this model, a transport equation is solved for $1 /(\gamma-1)$ in the non-conservative (advection) form. The mass and volume fractions can be recovered from $1 /(\gamma-1)$ by using the following equations:

$$
\begin{gathered}
\frac{1}{M}=\frac{Y_{1}}{M_{1}}+\frac{Y_{2}}{M_{2}} \\
\frac{1}{\gamma-1}=\frac{Y_{1}}{\gamma_{1}-1} \frac{M}{M_{1}}+\frac{Y_{2}}{\gamma_{2}-1} \frac{M}{M_{2}} \\
Y_{2}=1-Y_{1} \\
z_{1}=\frac{Y_{1} M_{2}}{Y_{2} M_{1}+Y_{1} M_{2}}
\end{gathered}
$$

where $M$ is the molecular mass, and $Y$ and $z$ are the mass and volume fraction of each species respectively.

The simulations were carried out using a finite volume MUSCL-Hancock shock-capturing scheme and highorder finite volume WENO schemes with Roe's approximate Riemann solver. ${ }^{19}$ The current interface-capturing formulation with variable $\gamma$ allows us to represent the correct transmitted and reflected shock speeds, time of reshock, and initial interface velocity, which was not the case in some prior works that used a single- $\gamma$ formulation. ${ }^{20}$

\section{Numerical simulation parameters}

The initial conditions for the present numerical simulations correspond to the $M a=1.21$ experiments of Collins and Jacobs performed in a vertical shock tube. ${ }^{16}$ In that work, a novel technique was used to generate the perturbed membrane-free gas-gas interface between air (acetone) mixture and sulfur hexafluoride $\left(\mathrm{SF}_{6}\right)$ gas and more resolved small features were captured by using planar laser-induced fluorescence (PLIF) flow visualization technique. The initial values are summarized in Table. 1. The post-shock Atwood number is 0.627 and the initial conditions across the shock wave are calculated by using Rankine-Hugoniot relations.

The current simulations were performed using a Fortran 90 code parallelized using MPI and run on the Nyx cluster at the Center for Advanced Computing at the University of Michigan. For each simulation, up to 160 processors were used. Three different grid resolutions corresponding to 148, 297 and 594 cells per wavelength (coarse, medium, fine) were used for the results presented in the next section. To represent the shock tube geometry, inflow boundary conditions are used at the entrance of the shock tube test section and reflecting boundary conditions are used along the remaining boundaries. 


\begin{tabular}{|l|l|l|l|l|l|}
\hline$\lambda(\mathrm{m})$ & 0.0594 & $x_{\text {interface }}(\mathrm{m})$ & 0.03 & $A$ & 0.605 \\
\hline$k(1 / \mathrm{m})$ & 105.77 & $t_{\text {reshock }}(\mathrm{s})$ & 0.0066 & $P(\mathrm{Pascal})$ & 95600 \\
\hline$a(\mathrm{~m})$ & 0.00183 & $u_{i, s h o c k}(\mathrm{~m} / \mathrm{s})$ & 370.98 & $M_{\text {air }}(\mathrm{g} / \mathrm{mol})$ & 34.76 \\
\hline$L x(\mathrm{~m})$ & 0.78 & $M a$ & 1.21 & $M_{S F 6}(\mathrm{~g} / \mathrm{mol})$ & 146.05 \\
\hline$L y(\mathrm{~m})$ & 0.0594 & $\rho_{\text {air }}\left(\mathrm{Kg} / \mathrm{m}^{3}\right)$ & 1.351 & $\gamma_{\text {air }}$ & 1.276 \\
\hline$x_{\text {shock }}(\mathrm{m})$ & 0.01 & $\rho_{S F 6}\left(\mathrm{Kg} / \mathrm{m}^{3}\right)$ & 5.494 & $\gamma_{S F 6}$ & 1.093 \\
\hline
\end{tabular}

Table 1. Summary of initial parameters of Collins and Jacob's shock tube experiments.

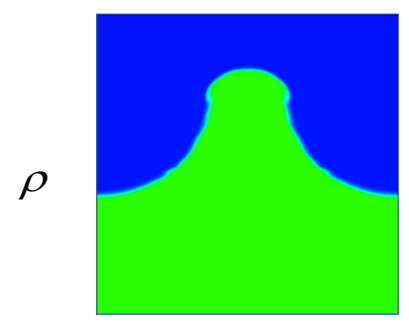

$2 \mathrm{~ms}$

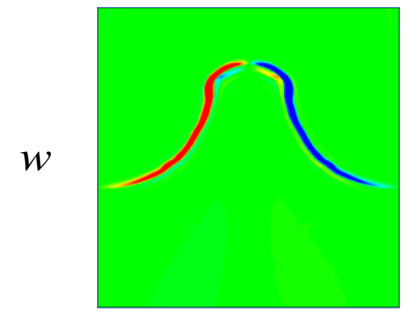

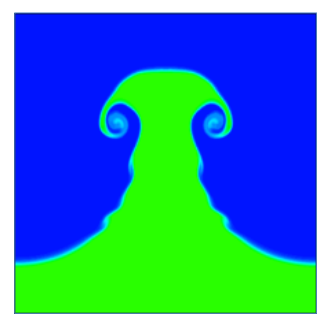

$4 \mathrm{~ms}$

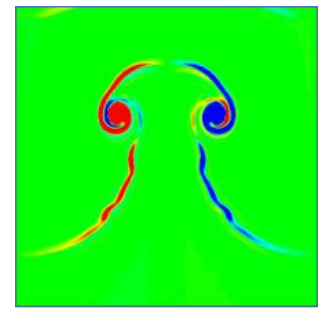

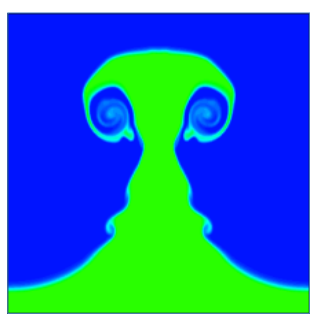

$6 \mathrm{~ms}$

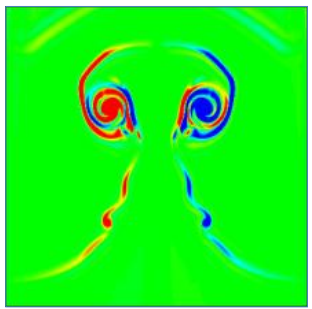

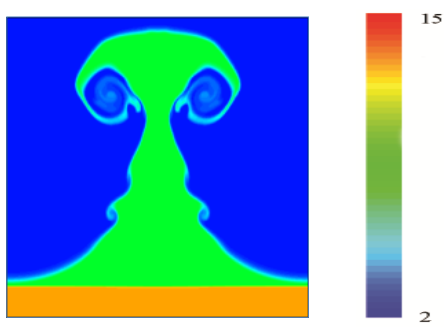

$6.6 \mathrm{~ms}$

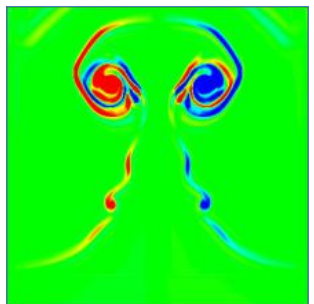

Figure 1. Density (top) and vorticity (bottom) after the passage of the initial shock. The shock came from top to bottom; air is on top, $\mathrm{SF}_{6}$ on the bottom. The mushroom-type structures characteristics of the $\mathrm{RM}$ instability develops.
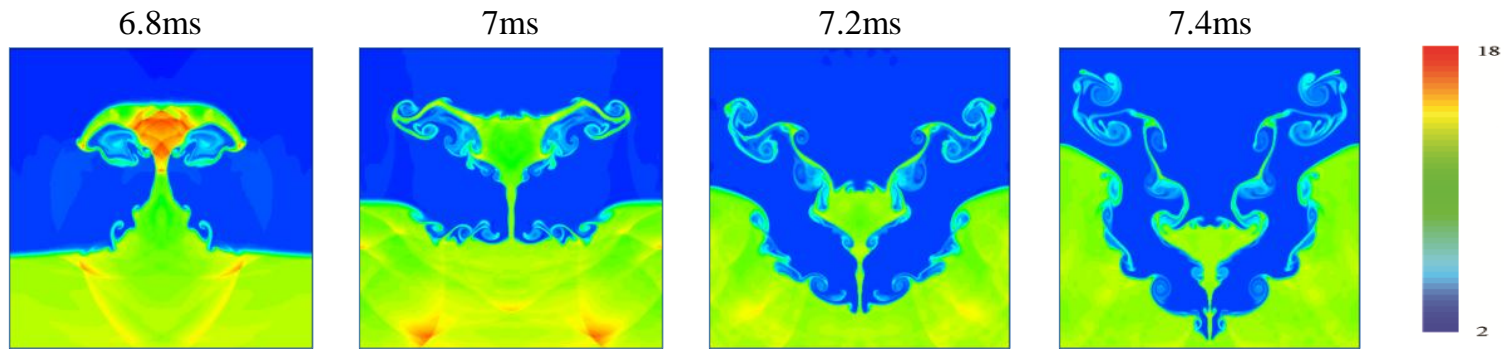

Figure 2. Density fields after reshock. The shock impacts the interface from the bottom and leads to the formation of small-scale features.

\section{Results and discussion}

\section{A. Qualitative analysis of density and vorticity evolution}

Fig. 1 represents the density and vorticity fields at 2.0, 4.0,6.0 and 6.6ms after the shock passes the interface. In the vorticity plots, the blue and red colors correspond to negative and positive vorticity. The mixing layer amplitude of the pre-shock perturbation decreases initially due to the compression of the incident shock wave. The Atwood number defined as $\left(\rho_{2}-\rho_{1}\right) /\left(\rho_{2}+\rho_{1}\right)$ is positive for the initial interaction. Consequently, the mixing layer amplitude starts to grow linearly, as predicted by impulsive models, and the interface keeps its sinusoidal shape until the amplitude-to-wavelength ratio reaches $0.1{ }^{3}$ At $2 \mathrm{~ms}$, the ratio of the amplitude to the wavelength is close to unity, which shows that non-linear effects have started affecting the flow. The heavier gas, $\mathrm{SF}_{6}$, starts to penetrate to the lighter air mixture while the vorticity distribution leads to roll-up and a mushroom structure at later times. A small layer with vorticity of the opposite sign can be seen close to the spike, which helps the roll-up process. At $4 \mathrm{~ms}$, the vorticity bi-layer can be seen in the vorticity plot. During the roll-up process, vorticity of opposite sign is generated due to the secondary baroclinic vorticity. ${ }^{9}$ The new vorticity distribution enhances the roll-up rate, thus 


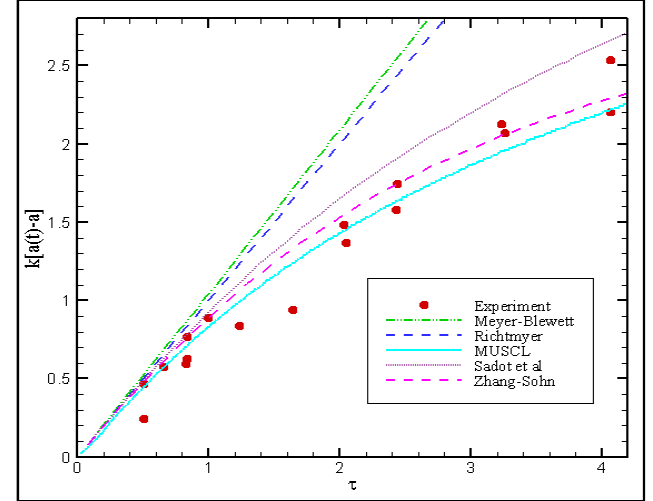

Figure 3. Time evolution of the dimensionless perturbation amplitude for different models.

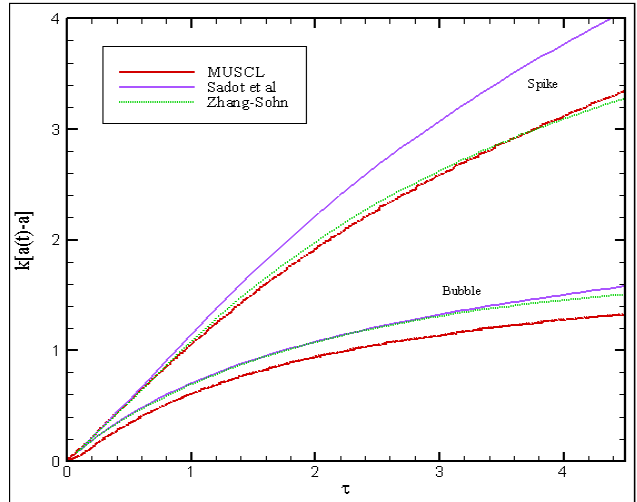

Figure 4. Time evolution of the bubble and spike dimensionless amplitude for different models.

resulting in the formation of more small scale features that can be seen in the density plots. As more $\mathrm{SF}_{6}$ penetrates the air mixture, the hat width of the mushroom structure increases while the neck thickness decreases.

At $6.6 \mathrm{~ms}$, reshock occurs. At this time, the interface profile is more complex compared to the initial sine wave and a vorticity distribution of different signs already exists along the interface. Hence, a greater rate of baroclinic vorticity is generated, compared to the first interaction between the shock wave and the interface. The Atwood number is negative for the $\mathrm{SF}_{6}$-air configuration, so that baroclinic vorticity, mostly of opposite sign, is deposited along the interface. After reshock, the neck thickness of the mushroom structure decreases more while new complex structures are generated at places where we have a close to planar interface at $6.8 \mathrm{~ms}$. The numerical dissipation of shock-capturing schemes affects the small-scale features appearing during and after the reshock process. By using either a higher-order accurate scheme or a higher resolution, we can minimize the numerical dissipation resulting in more small-scale features in the density plots. This can be seen in Fig. 2, which also results in a higher increase in the total mixing rate.

\section{B. Amplitude growth rate analysis}

Impulsive models for the amplitude growth rate such as the Richtmyer model and the Meyer-Blewett model are derived by substituting the constant acceleration in the Rayleigh-Taylor instability models with an impulsive acceleration. ${ }^{21}$ These models predict a constant amplitude growth rate which is applicable only for very early times that the flow is still in the linear regime. These velocities are used for scaling purposes. The non-dimensional time $\tau$ is defined as $\tau=k \dot{\mathrm{a}}_{0}^{+} t$ where $k$ is the wave number and $\dot{\mathrm{a}}_{0}^{+}=k A^{+} \mathrm{a}_{0}^{+} \Delta u$ is the post-shock Richtmyer velocity where $A^{+}$ is the post shock Atwood number, $\mathrm{a}_{0}^{+}$is the post shock amplitude and $\Delta u$ is the velocity of the unperturbed interface. Perturbation models such as the Zhang-Sohn model ${ }^{22}$ using a Pade expansion approximation and the Sadot et al model ${ }^{23}$ which are based on the asymptotic expansion of the linear perturbation equations make a very good agreement with the experimental results. The Zhang-Sohn model does not predict the 1/t asymptotic growth rate at late times while the Sadot et al model predicts the correct growth $1 / t$ asymptotic growth rate. In Fig. 3, MUSCL simulation on the fine grid and experimental data from Collins and Jacobs are compared with the predictions of models. All the models predict a similar behavior in the linear regime untill $\tau=0.7$. The Sadot et al model predicts a higher amplitude growth rate than the Zhang-Sohn model. Comparisons between bubble and spike amplitudes are also presented in Fig. 4. Our results for different schemes on the resolutions used here show good agreement with the experimental results for all of the cases.

\section{Circulation deposition on the perturbed interface}

The interaction of the shock and the interface results in vorticity deposition along the interface with maximum values at the spikes and minimum values at the bubbles. For sufficiently small perturbation amplitude, linear stability theory for incompressible flows can be used to evaluate the strength of the vortex sheet in the linear stage. ${ }^{24}$ The vorticity distribution can be written as

$$
\Omega(y)=2 \dot{a} \sin k y
$$

Integrating (8) over one half wavelength yields 


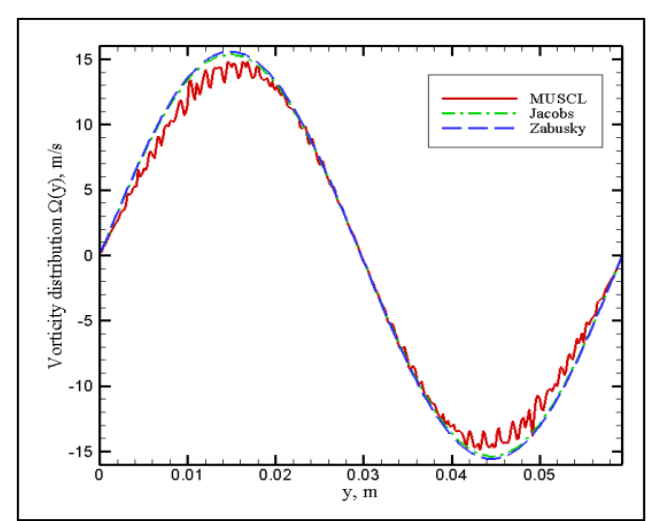

Figure 5. The initial baroclinic vorticity distribution along the interface by the incident shock.

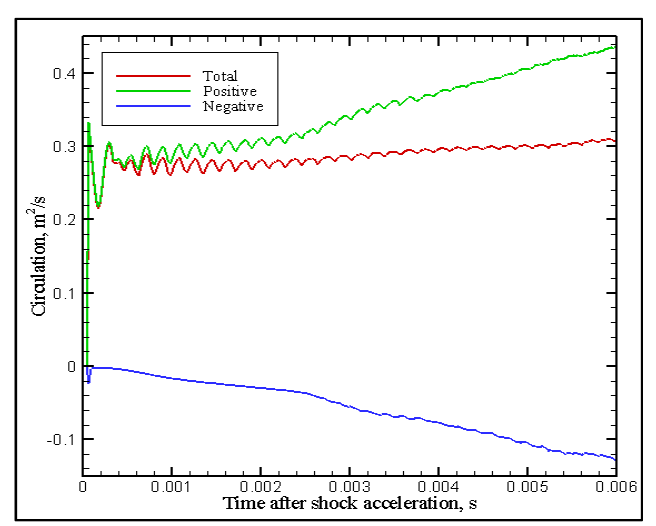

Figure 6. The time evolution of the positive, negative and total circulation just after the initial shock interaction.

$$
\Gamma=\int_{0}^{\pi / k} \Omega(y) d y=\frac{4}{k} \dot{a}_{0}^{+}
$$

In another approach, Samtaney and Zabusky used shock polar analysis to calculate the circulation deposition on shock-accelerated interfaces. ${ }^{25}$ Retaining only the first-order term, the non-dimensionalized vorticity distribution becomes

$$
\Omega(y)=\Gamma_{1}^{\prime} a_{0}^{+} k \sin k y
$$

where

$$
\Gamma_{1}^{\prime}=\frac{2 \gamma^{1 / 2}}{1+\gamma}\left(1-\eta^{-1 / 2}\right)\left(1-M a^{-1}+2 M a^{-2}\right)(M a-1), \text { and } \eta=\frac{\rho_{2}}{\rho_{1}}
$$

The vorticity field can be extracted numerically from the velocity field by putting a box around the interface. ${ }^{9}$ The vorticity distribution can be calculated from (12).

$$
\Omega(y)=\int_{x_{\text {spike }}-\Delta}^{x_{\text {buble }}+\Delta} \omega(x, y) d x
$$

Circulation then can be calculated by integrating the vorticity distribution. The total circulation is the summation of vorticity with positive and negative values in each cell. Fig.5 shows a comparison between the vorticity distributions of our simulations with the analytical models.

Following the passage of the shock wave, there is a jump in the circulation due to the baroclinic vorticity generation. The total circulation increases due to the secondary baroclinic vorticity generation until reshock happens. ${ }^{9}$ The total, positive and negative circulation over the interface are plotted in Fig. 6 . The absolute value of the negative circulation is a good representative of the strength of the bi-layer vorticity with opposite sign. The circulation plots also show that the present schemes deposit an adequate initial amount of vorticity along the interface.

\section{Integral parameters}

The instantaneous average of an arbitrary field $f(x, y, t)$ over the spanwise direction $y$ is defined as

$$
\langle f(x, t)\rangle=\frac{1}{L_{y}} \int f(x, y, t) d y
$$

The first integral parameter considered here is the total kinetic energy of the small scales $(K E)$. At each cross section, the mean velocities, $\hat{u}$ and $\hat{v}$, are subtracted from the pointwise velocities to calculate the pointwise velocity fluctuations. The average $K E$ for each section, $k$, is obtained by using (15). The $K E$ can be obtained by integrating $k$ over the whole domain.

$$
\begin{gathered}
\hat{u}=\langle\rho u\rangle /\langle\rho\rangle, \quad \hat{v}=\langle\rho v\rangle /\langle\rho\rangle \\
k=\left\langle\frac{1}{2} \rho\left((u-\hat{u})^{2}+(v-\hat{v})^{2}\right)\right\rangle /\langle\rho\rangle
\end{gathered}
$$

American Institute of Aeronautics and Astronautics 

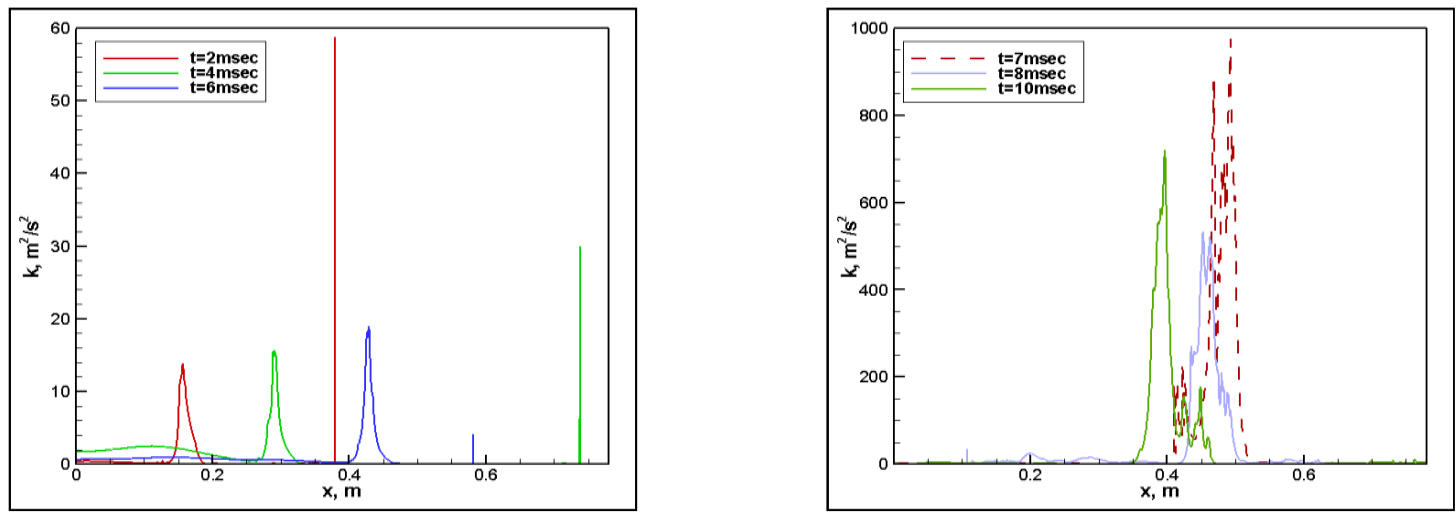

Figure 7. The average spanwise kinetic energy of small scales, $k(x)$, before (right) and after (left) reshock
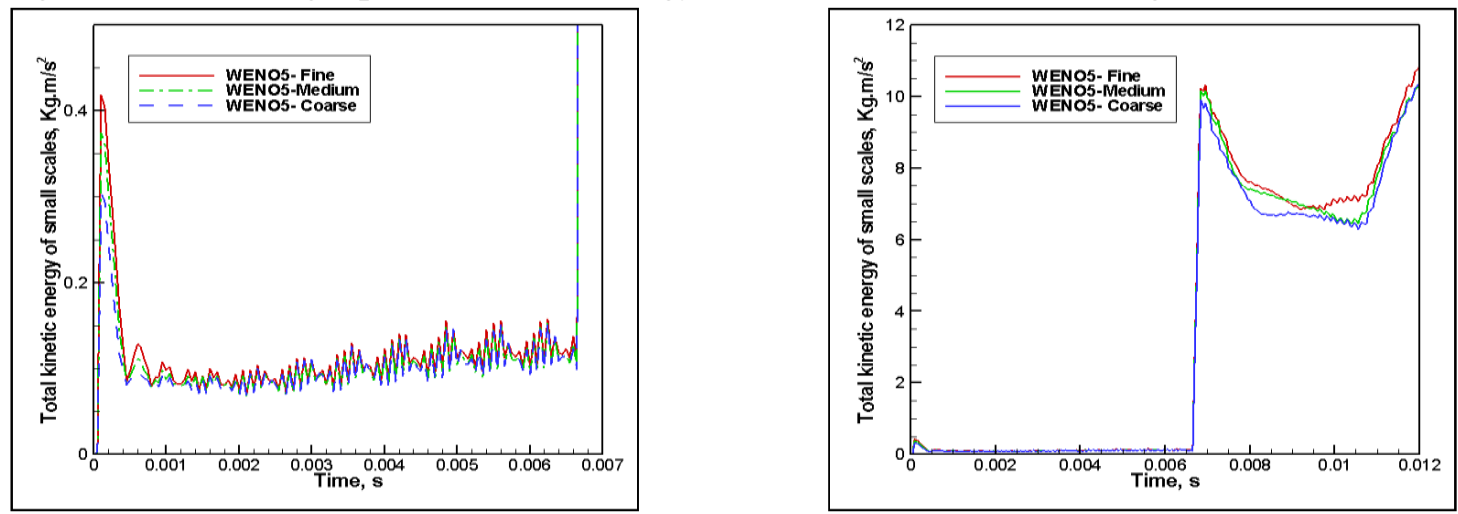

Figure 8. Evolution of total kinetic energv of small scales. $K E$. versus time.

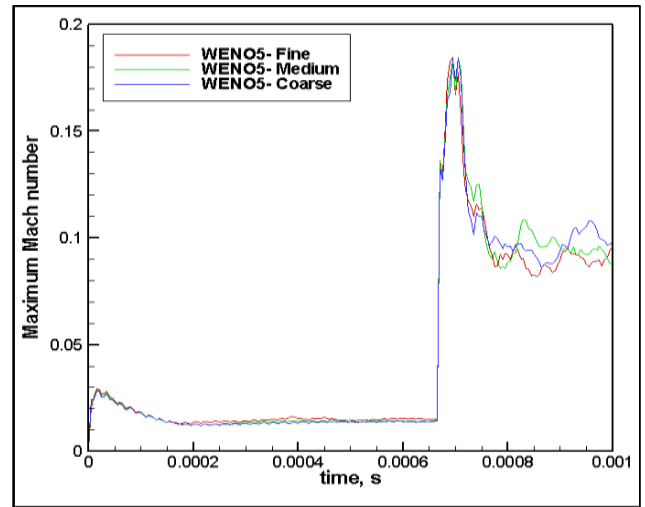

Figure 9. Maximum Mach number of the small scales versus time.

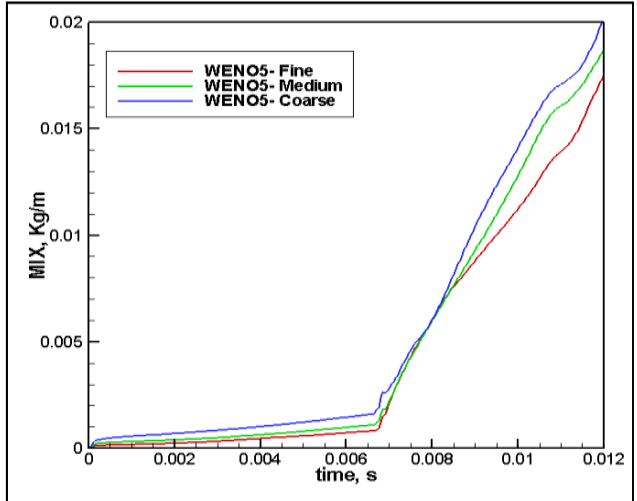

Figure 10. The time evolution of the total mixing.

$$
K E=\int\langle\rho\rangle k d x
$$

After the first interaction between the incident shock and the interface, a jump is observed in the $K E$. As expected, a larger amount of $K E$ is produced on the fine grid and remains almost constant until reshock. At the reshock, $K E$ increases dramatically by two orders of magnitude. Although the $K E$ reaches almost the same peak on both of the medium and fine grids, the decay rate is higher on the medium grid due to larger numerical dissipation.

The Mach number based on the velocity fluctuation of the small scales at each cross section, $M_{t}$, is defined as the ratio of the turbulent intensity, $u^{\prime}$, to the average of sound speed, $c$, where

$$
u^{\prime}=\sqrt{k}, \quad c=\langle\gamma \cdot p / \rho\rangle, \text { and } M_{t}=\frac{u^{\prime}}{c}
$$

Fig.9. shows the evolution of the maximum Mach number of the small scales versus time. Higher values during the interaction times suggest that compressibility effects are more dominant at these stages. Before the reshock, 

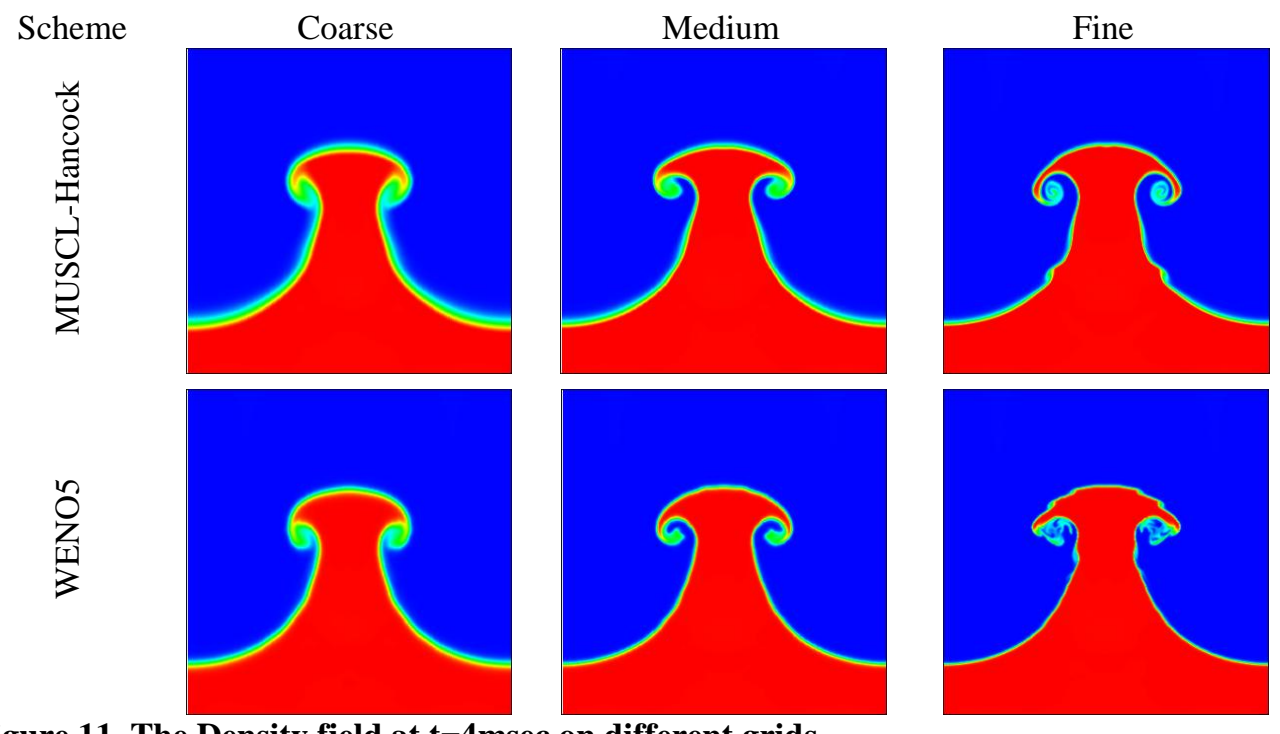

Figure 11. The Density field at $\mathrm{t}=4 \mathrm{msec}$ on different grids.
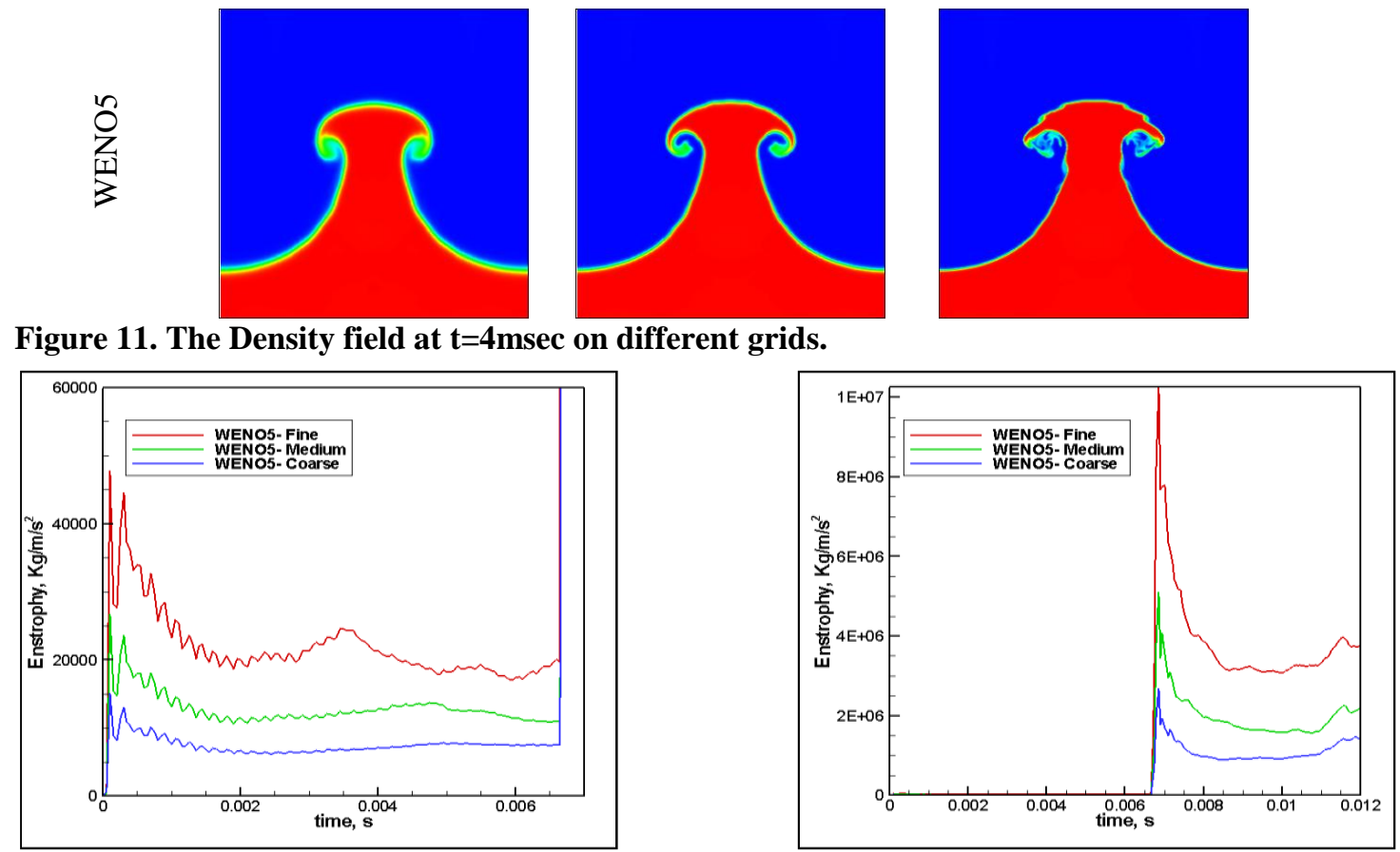

Figure 12. The time evolution of enstrophy versus time using WENO5 on different grids.

compressibility effects in the turbulence are not large for the current simulation. The $K E$ and $M_{t}$ defined above in the current two-dimensional simulations correspond to the turbulent kinetic energy and turbulent Mach number of the flow in a full three-dimensional simulation. Since the present simulations are two-dimensional, these values are interpreted as the kinetic energy and Mach number of the small scales

The second integral parameter considered here is the total mixing, MIX, defined as

$$
M I X=\int \rho^{2} Y_{1} Y_{2} d x d y
$$

The above equation can be thought of as the total reaction rate in a hypothetical chemical reaction between fluid 1 and fluid 2 where the reaction rate constant does not vary with temperature. ${ }^{26}$ In interface-capturing methods, a material interface between fluids 1 and 2 is smeared along a number of cells similarly to a shock in shock-capturing schemes. By using either a higher-order scheme or a finer grid, less numerical dissipation is added, thus resulting in a sharper interface/shock profile. The time evolution of the MIX is shown in Fig. 10. The more diffuse material interface profile on the coarse grid results in a higher value of the MIX until the reshock. The presence of more small-scale features on the fine grid before the reshock results in a higher rate of increase in the MIX for the fine grid after the reshock. At later times, the rate of increase in the MIX on the fine grid decreases again because of the role of numerical dissipation as discussed above. In the Richtmyer-Meshkov instability, mixing is coupled to the dynamics by homogenizing the density variations across the interface, thus affecting the baroclinic vorticity generation mechanism. In the present work, physical diffusion of mass is neglected, but grid-dependent numerical diffusion is added. Thus, MIX is a measure of entrainment and dispersion, and some numerical diffusion of mass. ${ }^{27}$

The last integral parameter considered here is the mass-weighted total enstrophy (ENS),

$$
E N S=\int \rho(\nabla \times u)^{2} d x d y
$$



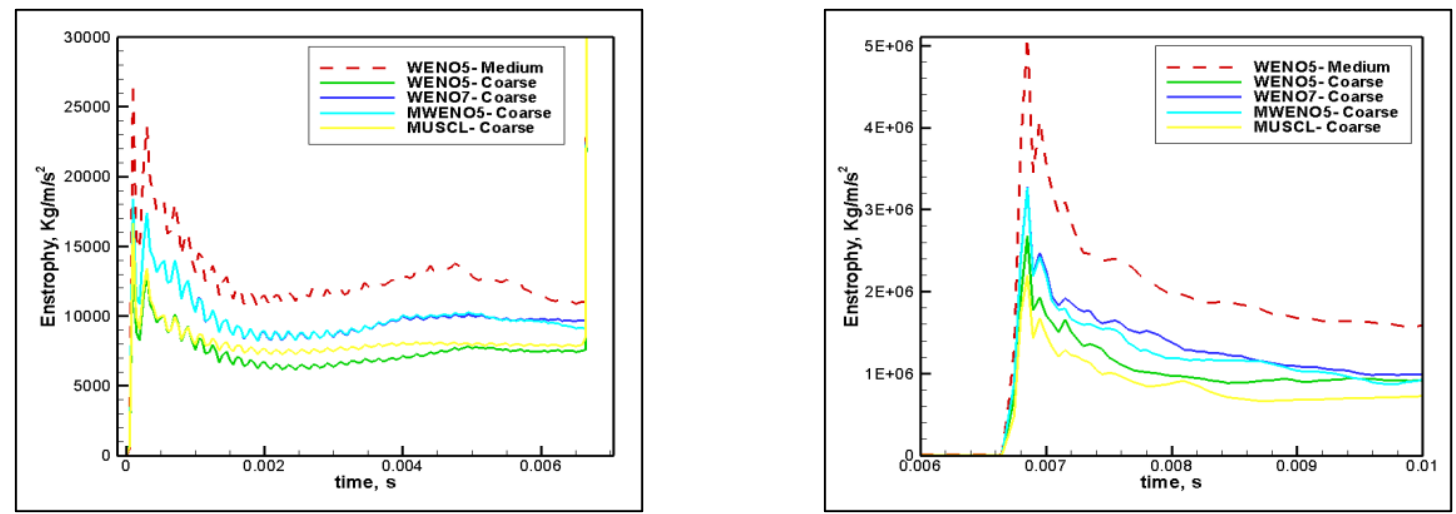

Figure 13. Comparison of the enstrophy evolution for different schemes.

\begin{tabular}{|l|c|c|c|c|}
\hline Scheme & MUSCL-Hancock & WENO5 & Mapped WENO5 & WENO7 \\
\hline Total CPU time(sec) & 1850 & 8500 & 9100 & 10500 \\
\hline Ratio & 1.00 & 4.59 & 4.92 & 5.67 \\
\hline
\end{tabular}

Table 2. Total CPU time for different schemes on the coarse grid until $0.05 \mathrm{msec}$.

As shown in Fig. 11, using either a high-order scheme or a fine grid results in more small-scale features with higher kinetic energy and vorticity. For the current problem, the enstrophy plots provide a good means to compare the efficiency of different schemes employed in this paper. The enstrophy evolution is presented in Fig. 12. Similarly to the KE and the total circulation plots, the enstrophy increases suddenly due to the initial interaction with the shock and after reshock.

\section{E. Comparison between the second-order MUSCL-Hancock and high-order WENO schemes}

The high-order accurate finite volume simulations of the Euler equations also require a high-order integral approximation of the fluxes at the interface. In order to satisfy this requirement, it is required to solve the Riemann problem twice at each interface at the Gaussian points and also to perform the WENO reconstruction in the both $x$ and $y$ directions to achieve high-order accuracy. This procedure makes the finite volume WENO approach approximately two times more expensive in two -dimensions and approximately four times more expensive in three -dimensions compared to the finite difference WENO approach.

In this paper, we have used a second-order MUSCL-Hancock scheme and several finite volume WENO schemes. ${ }^{11}$ Standard fifth-order and seventh-order accurate WENO schemes of Jiang and $\mathrm{Shu}^{13}$ and a fifth-order accurate mapped $\mathrm{WENO}^{14}$ scheme is used for comparison. It is worthwhile to mention that the seventh-order finite volume WENO is implemented with two Guassian points ${ }^{15}$, so that is really at best fourth-order accurate but with better resolution properties. The advantage of using a second-order scheme for the finite volume approach is that the Riemann problem is solved only once at each cell edge and the reconstruction is performed only once in either the $x$ or $y$ direction.

A comparison between the total CPU time and amount of numerical dissipation added by each scheme on the coarse grid quantified by means of enstrophy is presented below. The CPU times are calculated by running all of the test cases until $0.05 \mathrm{msec}$ on the coarse grid and are normalized in the table with respect to the MUSCL-Hancock results. The results show that mapped WENO5 and WENO7 results are very close to each other and are superior to the MUSCL-Hancock and WENO5 results. Considering that, in a two-dimensional calculation, reducing the grid size by a factor of two will approximately increase the total CPU time by a factor of eight and Table. 2, our results suggest that Mapped WENO5 is more efficient than WENO5 and even WENO7.

\section{F. The Euler versus Navier-Stokes simulations of shock dominated problems}

Shock-capturing schemes are capable of representing a shock wave on a grid by adding numerical dissipation. The amount of numerical dissipation scales with the grid size resulting in a sharper shock profile on a finer grid. As discussed in the previous section, as the grid is refined, more small-scale features appear before reshock, thus resulting in different flow behavior at late times. In nature, physical viscosity introduces a cut-off length scale, which means that for true direct numerical simulation (DNS) of these problems the grid size should have the same order of magnitude as this length scale. At these scales, physical viscosity should be dominant compared to the numerical dissipation. Thus, viscosity will determine the amount of small-scale features generated in the mixing region. 

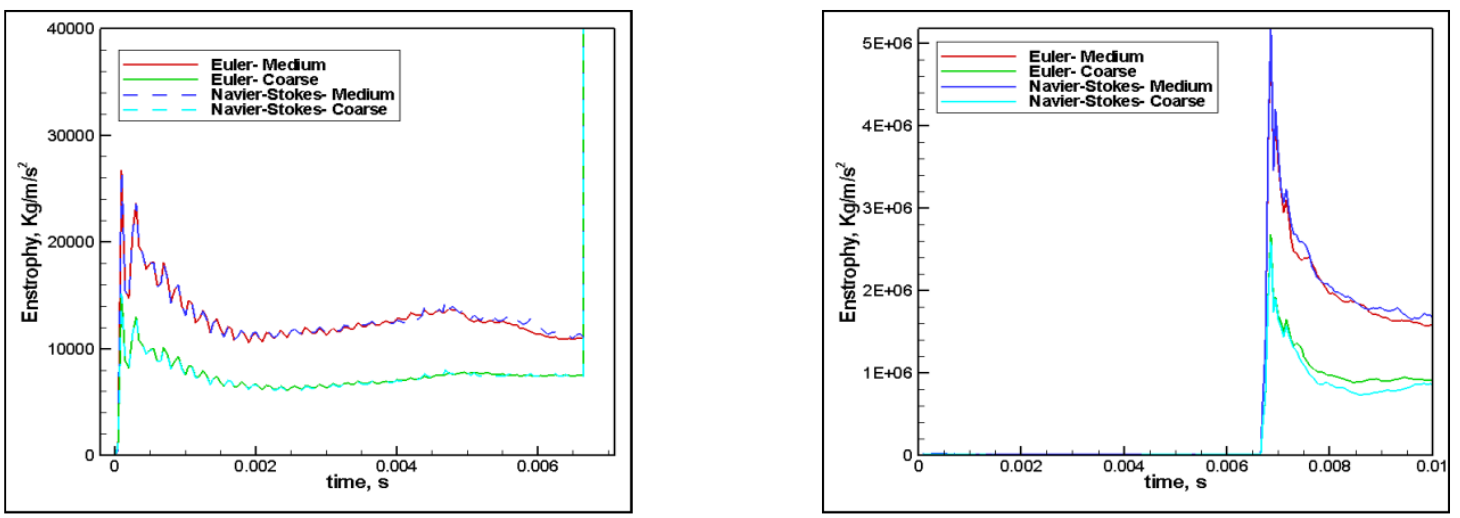

Figure 14. Comparison of the enstrophy evolution between the Euler and Navier-Stokes simulations with the actual diffusion coefficients.
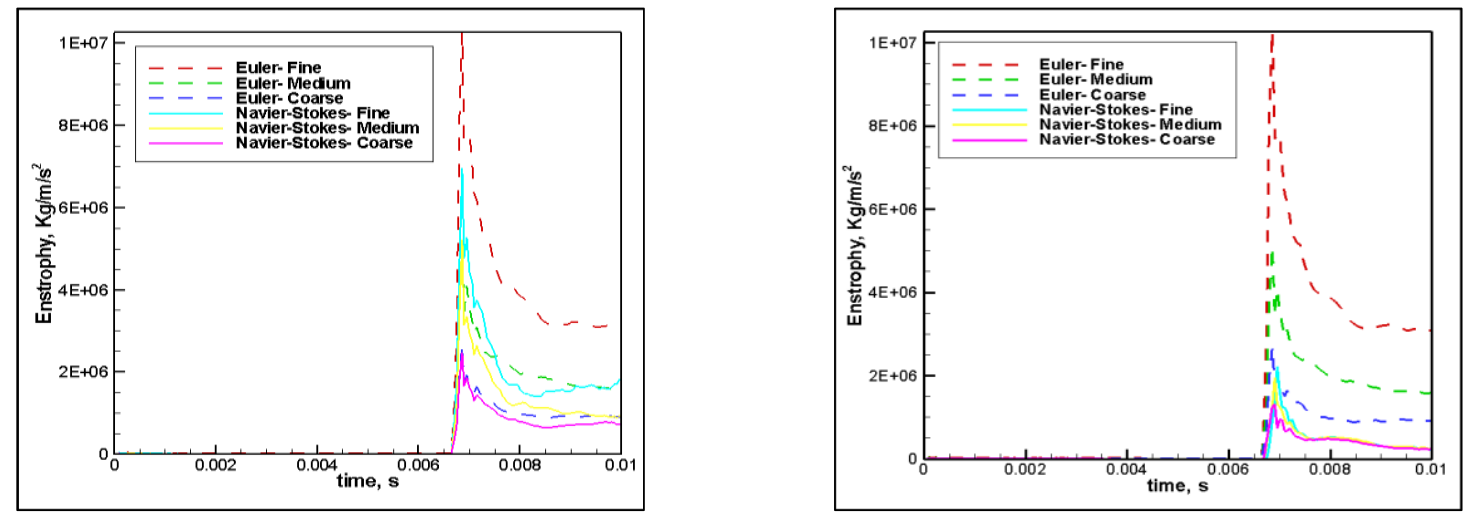

Figure 15. Comparison of the enstrophy evolution between the Euler and Navier-Stokes simulations with two (left) and three (right) orders of magnitude times the actual diffusion coefficients.

In order to investigate the role of viscosity and thermal conduction, the Navier-Stokes equations are solved with viscous and heat diffusion as diffusion fluxes. ${ }^{28}$ Mass diffusion is ignored. The heat conduction and viscosity coefficients of the mixture are determined from Herning and Zipper approximation for binary mixing and the temperature dependent pure component transport properties. ${ }^{29}$ As discussed in the previous sections, the enstrophy is a good measurement of the amount of small-scale features present at each time. For a converged solution, we expect the plots of enstrophy to lie on top of each other.

First, series of runs are performed with the diffusion coefficients corresponding to the actual diffusion coefficients of air and $\mathrm{SF}_{6}$ and the results are presented in Fig. 14. Effects of the diffusion terms are negligible on the results obtained before reshock on the grids used in the current work. After reshock, results are slightly different which shows that adding the diffusion terms could affect the results after reshock. Although some of the integral parameters show converging behavior, Fig. 14 suggests that we are far from the cut-off scale, as expected due to the high Reynolds number.

Second, the Reynolds number is decreased artificially by increasing the diffusion coefficients by two and three orders of magnitude. The main purpose is to determine the cut-off length scale and observe a converged behavior on an affordable grid. Fig. 15 shows a comparison of the enstrophy evolution between the Euler and Navier-Stokes simulations. Results show that increasing the diffusion coefficients will result in a decrease in the amount of difference in enstrophy generation on the coarse and medium grids. The plots for the lowest Reynolds number case that corresponds to increasing the diffusion coefficients by three orders of magnitude almost lie on top of each other which shows the converged behavior discussed above. The enstrophy plots of the Euler and Naveir-Stokes calculations with two orders of magnitude smaller Reynolds number on the coarse grid almost lie on top of each other. The viscous simulation on the medium grid shows less increase in enstrophy after reshock compared to the Euler simulation, illustrating the fact that the smallest scales generated by the Euler calculations may be unphysical. 


\section{Conclusion}

The development of the Richtmyer-Meshkov instability has been studied numerically for the single mode, Mach 1.21 air(acetone) $/ \mathrm{SF}_{6}$ shock tube experiment of Collins and Jacobs. ${ }^{16}$ The amplitude measurements from these simulations are in good agreement with analytical models and experimental data. The rate of baroclinic vorticity generation and its role in the evolution of the perturbed interface are also investigated. By using a $\gamma$-based model for preventing the spurious pressure oscillations at the interface, we were able to capture the correct transmitted and reflected shock speeds, time of reshock, and initial interface velocity comparing to other single- $\gamma$ numerical simulations.

For the current simulations, a converged behavior of the amplitude growth rate, total circulation and $K E$ were observed, specifically before reshock, with the schemes and resolutions employed. A CPU time and numerical dissipation analysis is performed and our conclusion is that Mapped WENO5 is more efficient compared to WENO5 and WENO7. The viscous and heat diffusion terms are added to achieve a converged solution and determine the DNS requirements for a given Reynolds number.

\section{Acknowledgments}

This research was supported in part by the DOE NNSA/ASC under the Predictive Science Academic Alliance Program by Grant No. DEFC52-08NA28616.

\section{References}

${ }^{1}$ Richtmyer, R.D., "Taylor Instability in Shock Acceleration of Compressible Fluids," Communications on Pure and Applied Mathmatics, Vol. 13, No. 13, 1960, pp. 297-319.

${ }^{2}$ Meshkov, E.E., "Instability of the Interface of Two Gases Accelerated by a Shock Wave," Fluid Dynamics, Vol. 4, 1969, pp. 101.

${ }^{3}$ Brouillette, M., "The Richtmyer-Meshkov Instability," Annual Review of Fluid Mechanics, Vol. 34, 2002, pp. 445-468.

${ }^{4}$ Taylor, G., "The Instability of Liquid Surfaces when Accelerated in a Direction Perpendicular to their Planes .1.," Proceedings of the Royal Society of London Series A-Mathematical and Physical Sciences, Vol. 201, No. 1065, 1950, pp. 192196.

${ }^{5}$ Drake, R.P., High-Energy-Density Physics, $1^{\text {st }}$ ed., Springer-Verlag, Berlin, 2006, Chap. 10.

${ }^{6}$ Yang, J., Kubota, T., and Zukoski, E., "Applications of Shock-Induced Mixing to Supersonic Combustion," AIAA Journal, Vol. 31, No. 5, May. 1993, pp. 854-862.

${ }^{7}$ Lindl, J.D., "Development of the Indirect-Drive Approach to Inertial Confinement Fusion and the Target Physics Basis for Ignition and Gain," Physics of Plasmas, Vol. 2, No. 11, Nov. 1995, pp. 3933-4024.

${ }^{8}$ Ryutov, D., Drake, R.P., Kane, J., Liang, E., Remington, B.A., and Wood-Vasey, W.M., "Similarity Criteria for the Laboratory Simulation of Supernova Hydrodynamics," Astrophysical Journal, Vol. 518, No. 2, 20 Jun. 1999, pp. 821-832.

${ }^{9}$ Aure, R., and Jacobs, J.W., "Particle Image Velocimetry Study of the Shock-Induced Single Mode Richtmyer-Meshkov Instability," Shock Waves, Vol. 18, No. 3, Aug. 2008, pp. 161-167.

${ }^{10}$ Hill, D.J., Pantano, C., and Pullin, D.I., "Large-Eddy Simulation and Multiscale Modeling of a Richtmyer-Meshkov Instability with Reshock," Journal of Fluid Mechanics, Vol. 557, 25 Jun. 2006, pp. 29-61.

${ }^{11}$ VanLeer, B., "Towards the Ultimate Difference Schemes .5. Second-Order Sequal to Godunov Method," Journal of Computational Physics, Vol. 32, No. 1, 1979, pp. 101-136.

${ }^{12}$ Shu, C.W., and Osher, S., "Efficient Implementation of Essentially Non-Oscillatory Shock-Capturing Schemes," Journal of Computational Physics, Vol. 77, No. 2, Aug. 1988, pp. 439-471.

${ }^{13}$ Jiang, G.S., and shu, C.W., "Efficient Implementation of Weighted ENO Schemes," Journal of Computational Physics, Vol. 126, No. 1, Jun. 1996, pp. 202-228.

${ }^{14}$ Henrick, A.K., Aslam, T.D., and Powers, J.M., "Mapped Weighted Essentially Non-Oscillatory Schemes: Achieving Optimal Order near Critical Points," Journal of Computational Physics, Vol. 207, No. 2, 10 Aug. 2005, pp. $542-567$.

${ }^{15}$ Titarev, V.A., and Toro, E.F., "Finite-Volume WENO Schemes for Three-dimensional Conservation Laws," Journal of Computational Physics, Vol. 201, No. 1, 20 Nov. 2004, pp. 238-260.

${ }^{16}$ Collins. B.D., and Jacobs, J.W., "PLIF Flow Visualization and Measurements of the Richtmyer-Meshkov Instability of an air/SF 6 Interface," Journal of Fluid Mechanics, Vol. 464, 10 Aug. 2002, pp. 113-136.

${ }^{17}$ Abgrall, R., "How to Prevent Pressure Oscillations in Multicomponent Flow Calculations: A Quasi Conservative Approach," Journal of Computational Physics, Vol. 125, No. 1, Apr. 1996, pp. 150-160.

${ }^{18}$ Saurel, R., and Abgrall, R., "A Simple Method for Compressible Multifluid Flows," SIAM Journal on Scientific Computing, Vol. 21, No. 3, 6 Dec. 1999, pp. 1115-1145.

${ }^{19}$ Roe, P.L., "Approximate Riemann Solvers, Parameter Vectors, and Difference-Schemes," Journal of Computational Physics, Vol. 43, No. 2, 1981, pp. 357-372.

${ }^{20}$ Latini, M., Schiling, O., and Don, W.S., "High-resolution Simulations and Modeling of Reshocked Single-mode Richtmyer-Meshkov Instability: Comparison to Experimental Data and to Amplitude Growth Model Predictions," Physics of Fluids, Vol. 19, No. 2, Feb. 2007, 024104. 
${ }^{21}$ Meyer, K.A., and Blewett, P.J., "Numerical Investigation of Stability of a Shock-Accelerated Interface between 2 Fluids," Physics of Fluids, Vol. 15, No. 3, 1972, pp. 753-759.

${ }^{22}$ Zhang, Q., Sohn, S.I, “Nonlinear Theory of Unstable Fluid Mixing Driven by Shock Wave,” Physics of Fluids, Vol. 9, No. 4, Apr. 1997, pp. 1106-1124.

${ }^{23}$ Sadot, O., Erez, L., Alon, U., Oren, D., and Levin, L.A., "Study of Nonlinear Evolution of Single-mode and Two-bubble Interaction under Richtmyer-Meshkov Instability," Physical Review Letters, Vol. 80, No. 8, 23 Feb. 1998, pp. $1654-1657$.

${ }^{24}$ Jacobs, J.W., and Sheeley, J.M., "Experimental Study of Incompressible Richtmyer-Meshkov Instability," Physics of Fluids, Vol. 8, No. 2, Feb. 1996, pp. 405-415.

${ }^{25}$ Samtaney, R., and Zabusky, N.J., "Circulation Deposition on Shock-accelerated Planar and Curved Density-stratified Interfaces - Models and Scaling Laws," Journal of Fluid Mechanics, Vol. 269, 25 Jun. 1994, pp. 45-78.

${ }^{26}$ Hahn, M., Drikakis, D., Youngs, D.L., and Williams, R.J.R, "Richtmyer-Meshkov Turbulent Mixing Arising from an Inclined Material Interface with Realistic Surface Perturbations and Reshocked Flow," Physics of Fluids, Vol. 23, No. 4, Apr. $2011,046101$.

${ }^{27}$ Dimotakis, P.E., “Turbulent Mixing,”Annual Review of Fluid Mechanics, Vol. 37, 2005, pp. 239-356.

${ }^{28}$ Toro, E.F., Riemann Solvers and Numerical Methods for Fluid Dynamics, $3^{\text {rd }}$ ed., Springer-Verlag, Berlin, 2009 , Chap. 1.

${ }^{29}$ Reid, R.C., Pransuitz, J.M., and Poling, B.E., The Properties of Gases and Liquids, $4^{\text {th }}$ ed., McGraw-Hill, Berlin, 1987, Chap. 9. 